\title{
Case Studies for Bearing Fault Diagnosis based on Adaptive Myriad Filter and Alpha Stable Model
}

\author{
Xinghui Zhang ${ }^{\mathrm{a}}$, Fei Zhao ${ }^{\mathrm{b}, \mathrm{c}, *}$ and Jianshe Kang ${ }^{\mathrm{a}}$ \\ ${ }^{a}$ Mechanical Engineering College, Shijiazhuang 050003, China \\ ${ }^{b}$ School of Business Administration, Northeastern University, Shenyang 110819, China \\ 'Northeastern University at Qinhuangdao, Qinhuangdao 066004, China
}

\begin{abstract}
Bearing fault diagnosis is a key research content of condition-based maintenance for machineries. Because of noise interference, incipient bearing fault is always difficult to be found. Traditionally, a large number of filtering algorithms used are limited to the cases of Gaussian noise or linear operation. In this paper, the adaptive Myriad filter and alpha stable model are elaborated. Myriad filter is a non-linear filter, which can be effectively applied in impulsive environment. The order of Myriad filter can be determined by alpha stable model. Finally, both laboratory fault data and real fault data from a public data set are used to verify the efficiency of the proposed method.
\end{abstract}

Keywords: Myriad filter; alpha stable model; bearing; fault diagnosis; non-linear filter

(Submitted on December 7, 2014; Revised on November 18, 2016; Accepted on April 23, 2017)

() 2017 Totem Publisher, Inc. All rights reserved.

\section{Introduction}

Though there are many theoretical achievements for condition based maintenance (CBM) [1,2], how to apply them into real engineering cases need further research. As a minor branch, bearing fault diagnosis attracts many scholars to investigate. A main problem of bearing fault diagnosis is the noise interference, which can enable the periodic impulsive signals (PIS) immersed. Therefore, scholars applied many filters to subtract noises unrelated to PIS. In these filters, non-linear filter such as morphological filter, median filter, etc are superior to the linear filters especially in real impulsive environments. Patargias et al [3] developed a morphological index to trend the defect level of rolling element bearings. Li et al [4] used morphological covering technique to diagnose the gear faults. Li et al [5] proposed a novel bearing fault diagnosis method using diagonal slice spectrum assisted optimal scale morphological filter. Meng et al [6] used morphological filter and invariant wavelet to denoise the bearing fault signal first. Then, revised ensemble empirical mode decomposition and envelope analysis were used to diagnose the bearing fault. Li et al [7] proposed a new fault diagnosis method for planetary gearbox based on empirical mode decomposition and adaptive multi-scale morphological gradient filter. In this paper, another non-linear filter named dynamic Myriad filter will be used for fault diagnosis. The filter order is determined by alpha stable model, which fitted to the bearing fault signal. Therefore, we say it is adaptive. This is also the main contribution of this paper. 


\section{Myriad Filter and Alpha Stable Model}

The Myriad filter can be given as follows: given a set of samples $x_{1}, x_{2}, \ldots, x_{N}$ and a real parameter $k>0$, the sample myriad of order $k$ is defined as [8]:

$$
\hat{\beta}_{k}=\operatorname{myriad}\left\{k ; x_{1}, \ldots, x_{N}\right\}=\arg \min _{\beta} \sum_{i=1}^{N} \log \left[k^{2}+\left(x_{i}-\beta\right)^{2}\right]
$$

Myriad filter is a running-window filter outputting the sample myriad of elements in the window. Actually, the sample myriad is the maximum likelihood estimator of location of a Cauchy distribution with semi-interquartile range $k$. This parameter plays an important role in fault diagnosis. When $k$ tends to infinity, the behavior of myriad filter closes to a linear estimator. In contrary, when it tends to zero, it will have good performance in the presence of very impulsive noise.

Under very general assumptions, the first order distribution of impulsive noise follows a symmetric alpha stable law [9]. The probability density function was defined as

$$
f_{\alpha}(\gamma, \delta ; x)=\frac{1}{2 \pi} \int_{-\infty}^{\infty} \exp \left(i \delta \omega-\gamma|\omega|^{\alpha}\right) e^{-i \omega x} d \omega
$$

Its three parameters are $\alpha$ (characteristic exponent, $0<\alpha<2), \gamma$ (dispersion, $\gamma>0$ ), and $\delta$ (location parameter, $-\infty<\delta<\infty)$. In reference [8], an empirical relation between Myriad filter order and parameters of alpha stable model was given

$$
k(\alpha)=\sqrt{\frac{\alpha}{2-\alpha}} \gamma^{1 / \alpha}
$$

Therefore, Myriad filter order $k$ can be determined by parameters of alpha stable model. And, these parameters are determined according to the signal. So, we say the filter is adaptive to the signal.

\section{Two Case Studies of Bearing Fault Diagnosis}

\subsection{Injected fault case study}

We used a public data set from [10] to verify the proposed method. The bearing geometric parameters are as follows: roller diameter 0.235 inch, pitch diameter 1.245 inch, number of elements is 8 , and the contact angle equal to $0^{\circ}$. The sample frequency is $48,828 \mathrm{~Hz}$ and lasting 3 seconds. The outer race of this bearing was injected fault. The input shaft rotation rate is $25 \mathrm{~Hz}$ and load is $25 \mathrm{lbs}$. If we define the four fault characteristic frequencies as ball pass frequency inner race (BPFI), ball pass frequency outer race (BPFO), ball spin frequency (BSF), and fundamental train frequency (FTF). They can be calculated according to geometric parameters of the bearing. They are: BPFO (81.12 Hz), BPFI (118.88 Hz), BSF (63.86 $\mathrm{Hz}$ ) and FTF (10.14 Hz). Four equations are as follows:

$$
\begin{gathered}
F_{\text {outer }}=\frac{N_{B}}{2} F_{R}\left(1-\frac{D_{B} \cos (\theta)}{D_{P}}\right) \\
F_{\text {inner }}=\frac{N_{B}}{2} F_{R}\left(1+\frac{D_{B} \cos (\theta)}{D_{P}}\right) \\
F_{\text {ball }}=\frac{D_{P}}{D_{B}} F_{R}\left(1-\frac{D_{B}{ }^{2} \cos ^{2}(\theta)}{D_{P}^{2}}\right)
\end{gathered}
$$




$$
F_{\text {cage }}=\frac{1}{2} F_{R}\left(1-\frac{D_{B} \cos (\theta)}{D_{P}}\right)
$$

In these equations, $F_{R}$ is the shaft speed, $N_{B}$ is the number of rolling element in bearing, $D_{B}$ is the ball diameter, $D_{P}$ is the pitch diameter, and $\theta$ is the ball contact angle. When the rotation speed is known, the fault frequencies can be calculated.

For Myriad filter, we can select two kinds of windows: one window is 10, the other is 100 . For window size 10, the denoise result can be shown in Figure 1. From this figure, we can see that some impulsive noises are subtracted. However, the PIS produced by bearing fault are reserved. For window size 100, the denoise result can be shown in Figure 2. It shows that the signals unrelated to the PIS produced by bearing fault are suppressed sufficiently. Compared to result of window size 10, it is more effective. Figure 3 is the envelope spectrum comparison of original signal, denoised signal by Myriad filter with window size 10, and denoised signal by Myriad filter with window size 100. From figure 3, we can see the obvious bearing outer race fault frequency BPFO in three envelope spectrums. However, the amplitude of envelope spectrum of denoised signal by Myriad filter with window size 100 is the biggest. Second is the amplitude of envelope spectrum of denoised signal by Myriad filter with window size 10. Therefore, we can say that adaptive Myriad filter can subtract the noise effectively and enable the bearing fault frequency obvious.
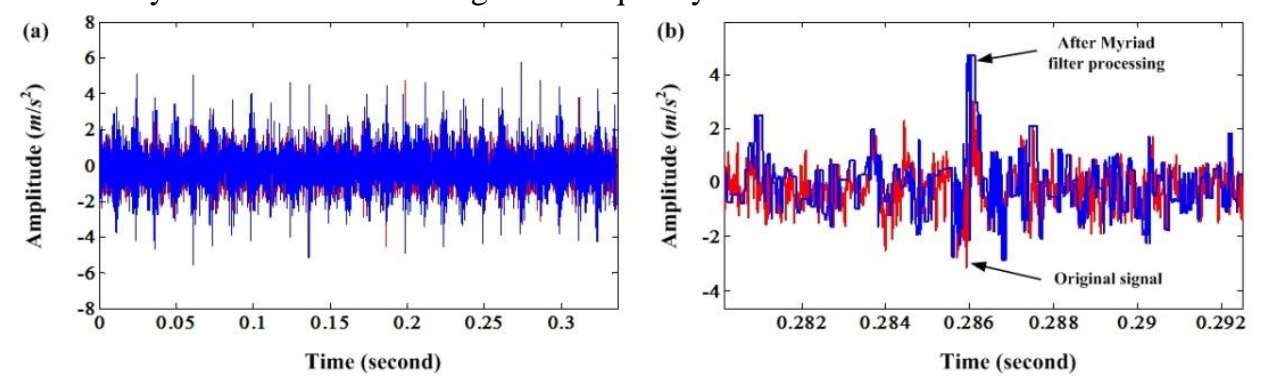

Figure 1. (a) Original signal and denoised signal by Myriad filter with window size 10; (b) Local amplify of (a)
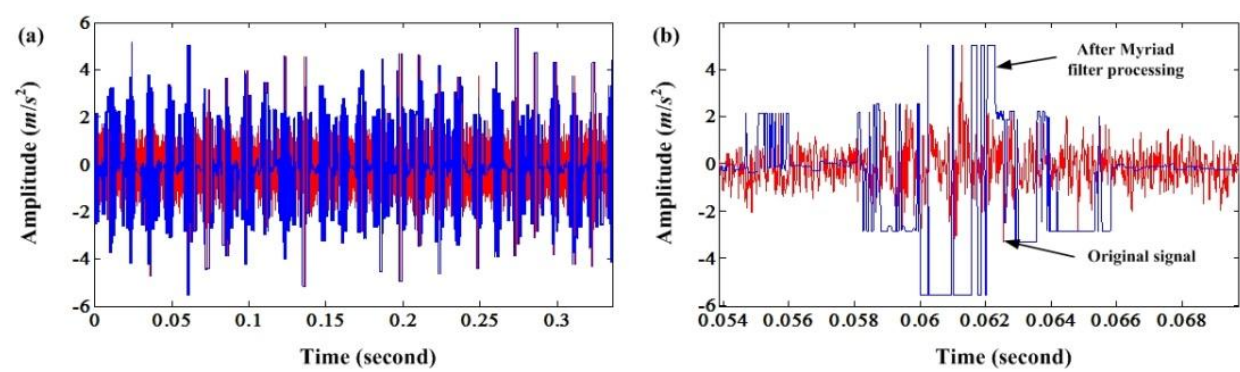

Figure 2. (a) Original signal and denoised signal by Myriad filter with window size 100; (b) Local amplify of (a)

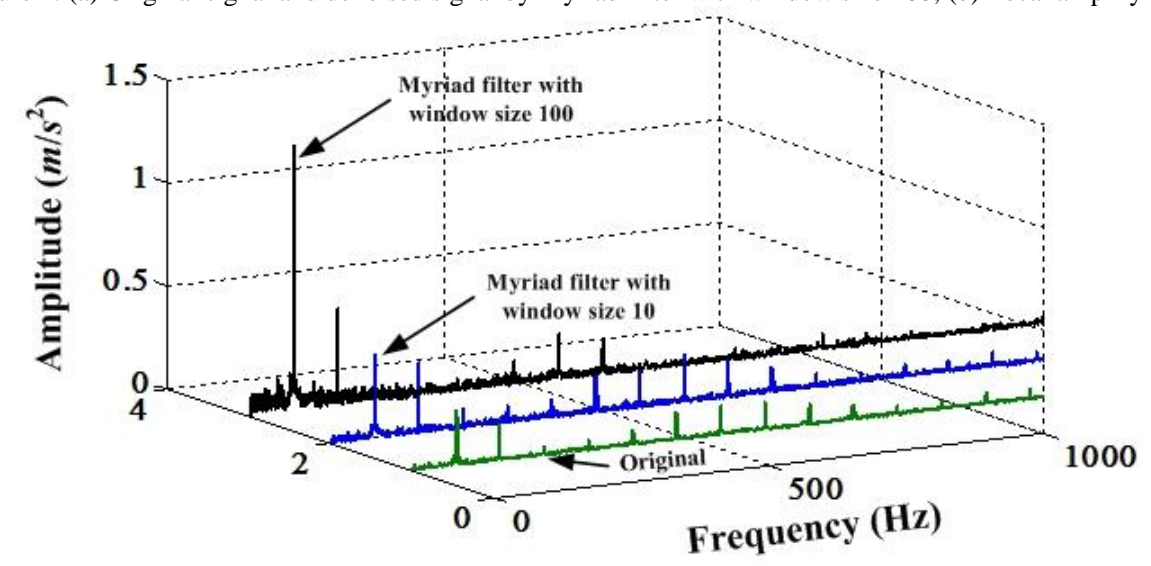

Figure 3. Envelope spectrums comparison of original signal and denoised signal using Myriad filter

In order to further demonstrate the effectiveness of proposed method, we use another non-linear filtering method (morphological filtering) to process same data for comparison. In this process, dilate-erode gradient was used and structure element was optimized by kurtosis. The envelope results can be seen in Figure 4. Compared with result of Myriad filter with 
window size 100, the amplitude of BPFO is slightly smaller. In other words, the local signal noise ratio of Myriad filter result is slightly higher than morphological filter result.

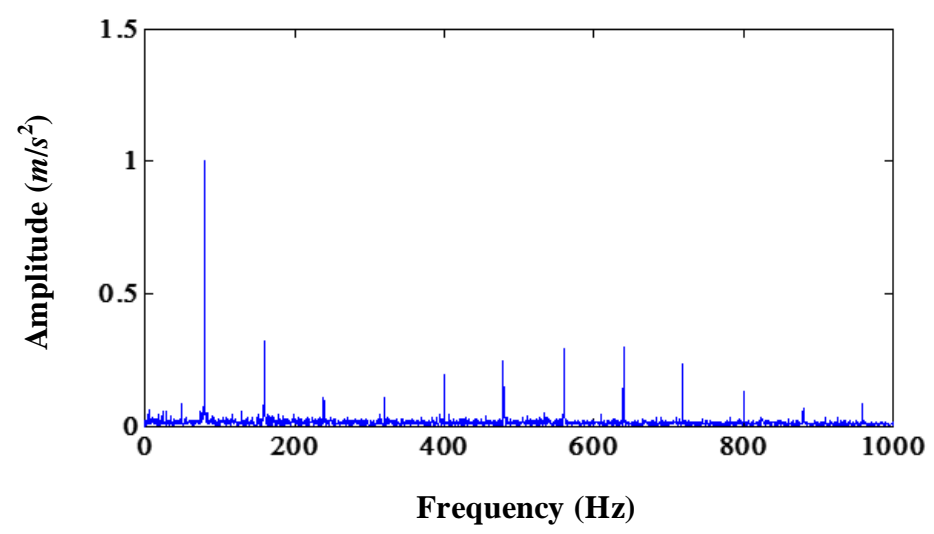

Figure 4. Envelope spectrum of signal processed by morphological filtering

\subsection{Real bearing fault of planetary gearbox case study}

This data is also come from public data sets [10]. It is a ball fault of intermediate shaft bearing of planetary gearbox. The four fault frequencies related to this bearing are: BPFO $(51.89 \mathrm{~Hz}), \mathrm{BPFI}(67.85 \mathrm{~Hz}), \mathrm{BSF}(24.3 \mathrm{~Hz})$ and FTF $(2.76 \mathrm{~Hz})$. For this case, only envelope spectrums comparison results are given as illustrated in Figure 5. It shows that envelope spectrum of denoised signal by Myriad filter with window size 20 has the best effect. Ball fault frequency and its harmonics are obvious and have biggest amplitude. However, the effect of envelope spectrum filtered by Myriad filter with window size 100 has not good effect. It is even worse than the envelope spectrum of original signal. From injected fault case study and real fault case study, we can see the window size of Myriad filter is an important factor to determine the performance of Myriad filter.
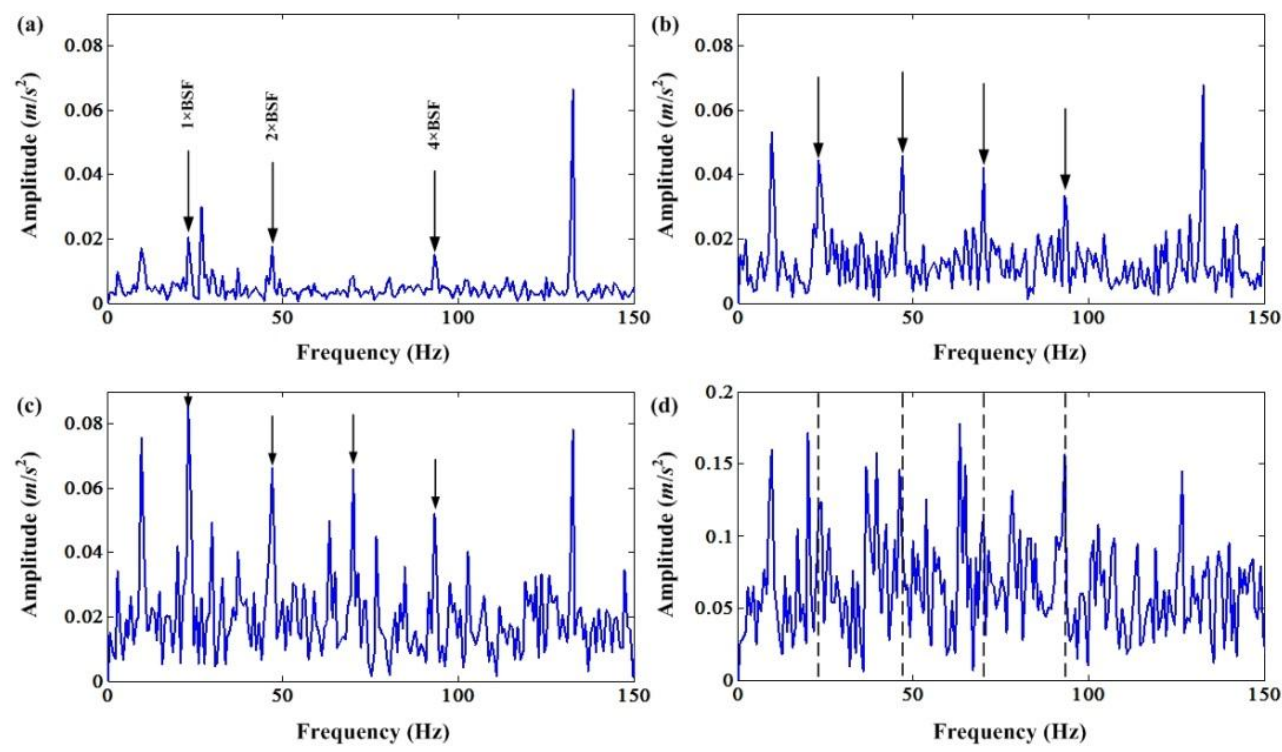

Figure 5. (a) Envelope spectrum of original signal; (b) Envelope spectrum of denoised signal by Myriad filter with window size 10; (c) Envelope spectrum of denoised signal by Myriad filter with window size 20; (d) Envelope spectrum of denoise signal by Myriad filter with window size 100

Similarly, the result processed by morphological filter can be seen in Figure 6. The dilate-erode gradient also was used in this processing and structure element was optimized by kurtosis. From this figure, we can see that there are many noise frequency in envelope spectrum though the second and fourth harmonic of BSF can be found. So, we can conclude that proposed method has a good effect for bearing fault diagnosis. 


\section{Conclusions}

In this paper, a bearing fault diagnosis method based on adaptive Myriad filter and alpha stable model is proposed. This adaptive filter can suppress the signal component unrelated to bearing fault effectively. Therefore, the PIS produced by bearing fault is enhanced and enable the fault diagnosis easier. Two case studies demonstrate the validity of proposed method.

\section{Acknowledgements}

The research is partially supported by the China NSFC under grant number 71401173, the Natural Science Foundation of Hebei Province under grant number E2015506012, China Ministry of Education Humanities and Social Sciences Research Youth Fund Project 16YJC630174, the Fundamental Research Funds for the Central Universities of China N152303004, Hebei Province High School Science and Technology Research Project QN2017104, Hebei Province Soft Science Research Planning Project 16457402D, the Fundamental Research Funds for Northeastern University at Qinhuangdao under grant number XNB201616.

\section{References}

1. A. K. S. Jardine, D. Lin, and D. Banjevic, "A Review on Machinery Diagnostics and Prognostics Implementing ConditionBased Maintenance," Mechanical Systems and Signal Processing, vol. 20, no. 7, pp. 1483-1510, October 2006.

2. T. C. Kumar, G. Singh, and V. N. A. Naikan, "Broken Rotor Bar Fault Diagnosis in VFD Driven Induction Motors by an Improved Vibration Monitoring Technique," International Journal of Performability Engineering, vol. 13, no. 1, pp. 87-94, January 2017.

3. T. I. Patargias, C. T. Yiakopoulos, and I. A. Antoniadis, "Performance Assessment of a Morphological Index in Fault Prediction and Trending of Defective Rolling Element Bearings," Nondestructive Testing and Evaluation, vol. 21, no. 1, pp. 39-60, August 2006.

4. B. Li, P. L. Zhang, Z. J. Wang, S. S. Mi, and P. Y. Liu, "Morphological Covering based Generalized Dimension for Gear Fault Diagnosis," Nonlinear Dynamics, vol. 67, no. 4, pp. 2561-2571, August 2012.

5. Y. F. Li, X. H. Liang, and M. J. Zuo, "Diagonal Slice Spectrum Assisted Optimal Morphological Filter for Rolling Element Bearing Fault Diagnosis," Mechanical Systems and Signal Processing, vol. 85, pp. 146-161, February 2017.

6. L. J. Meng, J. W. Xiang, Y. X. Wang, Y. Y. Jiang, and H. F. Gao, "A Hybrid Fault Diagnosis Method using Morphological Filter-Translation Invariant Wavelet and Improved Ensemble Empirical Mode Decomposition," Mechanical Systems and Signal Processing, vol. 50-51, pp. 101-115, January 2015.

7. H. P. Li, J. M. Zhao, X. H. Zhang, and X. L. Ni, "A New Fault Diagnosis Method for Planetary Gearbox," International Journal of Performability Engineering, vol. 12, no. 4, pp. 389-394, July 2016.

8. J. G. Gonzalez and G. R. Arce, "Optimality of the Myriad Filter in Practical Impulsive-Noise Environments," IEEE Transactions on Signal Processing, vol. 49, no.2, pp. 438-441, February 2001.

9. G. A. Tsihrintzis and C. L. Nikias, "Fast Estimation of the Parameters of Alpha-Stable Impulsive Interference," IEEE Transactions on Signal Processing, vol. 44, no. 6, pp. 1492-1503, June 1996.

10. Fault Data Sets: http://www.mfpt.org/. Accessed 2013. 